Claremont Colleges

Scholarship@ Claremont

All HMC Faculty Publications and Research

HMC Faculty Scholarship

4-1-2002

\title{
Four-Person Envy-Free Chore Division
}

Elisha Peterson '00

Harvey Mudd College

Francis E. Su

Harvey Mudd College

\section{Recommended Citation}

Elisha Peterson and Francis Edward Su. Four-Person Envy-Free Chore Division. Math. Mag., 75(2):117-122, 2002.

This Article is brought to you for free and open access by the HMC Faculty Scholarship at Scholarship @ Claremont. It has been accepted for inclusion in All HMC Faculty Publications and Research by an authorized administrator of Scholarship @ Claremont. For more information, please contact scholarship@cuc.claremont.edu. 


\title{
Four-Person Envy-Free Chore Division
}

\author{
ELISHA PETERSON \\ Magdalen College, Oxford University \\ Oxford OX1 4AU, U.K. \\ FRANCIS EDWARD SU \\ Harvey Mudd College \\ Claremont, CA 91711
}

In this article we explore the problem of chore division, which is closely related to a classical question, due to Steinhaus [10], of how to cut a cake fairly. We focus on constructive solutions, that is, those obtained via a well-defined procedure or algorithm. Among the many notions of fairness is envy-freeness: an envy-free cake division is a set of cuts and an allocation of the pieces that gives each person what she feels is the largest piece. It is non-trivial to find such a division, since the cake may not be homogeneous and player valuations on subsets of cake will differ, in general. Much progress has been made on finding constructive algorithms for achieving envy-free cake divisions; most recently, Brams and Taylor [3] produced the first general $n$-person procedure. The recent books by Brams and Taylor [4] and Robertson and Webb [8] give surveys on the cake-cutting literature.

In contrast to cakes, which are desirable, the dual problem of chore division is concerned with dividing an object deemed undesirable. Here, each player would like to receive what he considers to be the smallest piece of, say, a set of chores. This problem appears to have been first introduced by Martin Gardner [6].

Much less work has been done to develop algorithms for chore division than for cake-cutting. Of course, for 2 people, the familiar I-cut-you-choose cake-cutting procedure also works for dividing chores: one cuts the chores and the other chooses what she feels is the smallest piece. Oskui [8, p. 73] gave the first envy-free solutions for chore division among 3 people. Su [12] developed an envy-free chore-division algorithm for an arbitrary number of players; however, it does not yield an exact solution, but only an $\epsilon$-approximate one. There appear to be no exact envy-free chore-division algorithms for more than three players in the literature; in unpublished manuscripts, Brams and Taylor [2] and Peterson and Su [7] offer $n$-person algorithms but these are not bounded in the number of steps they require.

In this article, we develop a simple and bounded procedure for envy-free chore division among 4 players. The reader will find that many of the ideas involved-moving knives, trimming and lumping, and a notion of "irrevocable advantage"-provide a nice introduction to similar techniques that arise in the literature on fair division problems. As a warm-up to some of these ideas, we also present a 3-person solution that is simpler and more symmetrical than the procedure of Oskui.

We assume throughout this paper that chores are infinitely divisible. This is not unreasonable, as a finite set of chores can be partitioned by dividing up each chore (for instance, a lawn to be mowed could be divided just as if it were a cake), or dividing the time spent on them. We also assume that player valuations over subsets of the chores 
are additive, that is, no value is destroyed or created by cutting or lumping pieces together. (The proper context for modeling player valuations is measure theory, but we can avoid that for the purposes of this article.)

A 3-person chore-division procedure We first describe a simpler 3-person choredivision procedure, which introduces some ideas that are important later.

Our 3-person chore-division procedure relies on Austin's procedure [1] for dividing a cake into two pieces so that each of two players believes it is a 50-50 split. For completeness, we review it here. Let one player hold two knives over the cake, with one at the left edge, such that the portion of cake between them is what she believes to be exactly half. If the second player agrees that it is exactly half, we are done. Otherwise, let the first player move the knives across the cake from left to right, keeping the portion between them exactly half (in her estimation), until the second player agrees it is exactly half. (There must be such a point because when the rightmost knife reaches the right edge, the leftmost knife must be where the rightmost knife began, hence the second player must by that point have changed preferences.) At this point cuts are made and the pieces of cake outside the knives are lumped together, yielding two pieces that both players agree are exactly equal.

Austin's procedure is an example of what is sometimes called a "moving-knife" procedure in the cake-cutting literature [5]. Our 3-person chore-division algorithm is also a moving-knife procedure. The key idea is to divide the chores into six pieces and assign each player two of the pieces that he feels are at least as small as each pair of pieces the other players receive.

\section{A THREE-PERSON ENVY-FREE CHORE-DIVISION PROCEDURE}

Step 1. Divide the chores into three portions using any 3-person envy-free cakedivision procedure (that guarantees players a piece they think is largest), such as the Stromquist moving-knife procedure [11]. Now label each portion by the name of the player to whom the cake-division procedure would assign that portion (this player believes that portion is largest).

Step 2. Let player $i$ divide portion $i$ into 2 pieces (which she feels is exactly half) and assign those pieces to the other two players such that they each feel they have received no more than half of portion $i$. (This can be achieved via Austin's procedure: letting player $i$ and one other player, say $j$, agree on a 50-50 split, let the remaining player choose the half she thinks is smallest, and give the other half to $j$.)

Step 3. Repeat Step 2 for each player, then end the procedure.

We now verify that each player has been assigned two out of six total pieces such that each feels her share is smallest.

Call the players $i, j$, and $k$. Player $i$ will not envy player $j$ because one piece of each of their pairs came from the portion labelled $k$, and $i$ feels her half of that portion was no larger than $j$ 's. As for her other piece, player $i$ feels it was no more than half of the portion it came from, and therefore cannot be as large as player $j$ 's other piece, which $i$ felt was exactly half of the largest portion. The same argument holds for any permutation of $i, j$, and $k$. See FIGURE 1 .

This procedure requires at most 8 cuts (Step 1 uses 2 cuts, and Austin's procedure uses at most 2 cuts each time it is applied; in FIGURE 1, some pieces may have been reassembled for simplicity). It is also less complicated than the discrete procedure of Oskui [8]. There are some 3-person moving-knife schemes that require fewer cuts $[8,9]$, but our approach is distinguished by being symmetric with respect to the players and being based on a cake-cutting procedure. The former property simplifies 


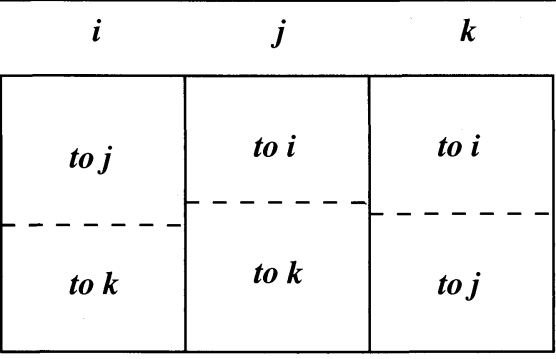

Figure 1 An envy-free assignment of six pieces (of chores) to three people

the verification of envy-freeness, while the latter property may help in generalizing the scheme to more players via known cake-cutting algorithms.

A 4-Person Chore-division Procedure We now describe our 4-person choredivision procedure, which is also a moving-knife procedure and requires at most 16 cuts. It draws ideas from both the Brams-Taylor-Zwicker 4-person envy-free movingknife scheme for cakes [5] and the Oskui 3-person envy-free discrete chore-division scheme [8]. We also show how the notion of irrevocable advantage, important in cake-cutting [4], can be applied in chore division.

Suppose the players are named Alice, Betty, Carl, and Debbie. For convenience, we assume the chores are a rectangular block that may be divided by vertical cuts. Let Alice and Betty divide the chores into four pieces they both agree are all equal, by performing three applications of Austin's procedure (using at most 6 cuts).

Call the pieces $X_{1}, X_{2}, X_{3}$, and $X_{4}$. Note that if Debbie and Carl disagree on which piece is the smallest, we can immediately allocate the pieces. Thus we may assume they agree that one piece is strictly smaller than the others, say $X_{4}$. Then each person thinks the following:

$$
\begin{aligned}
\text { Alice: } & X_{1}=X_{2}=X_{3}=X_{4} \\
\text { Betty: } & X_{1}=X_{2}=X_{3}=X_{4} \\
\text { Carl: } & X_{4}<X_{1}, X_{2}, X_{3} \\
\text { Debbie: } & X_{4}<X_{1}, X_{2}, X_{3} .
\end{aligned}
$$

Now, for each of $X_{1}, X_{2}$, and $X_{3}$, let Debbie and Carl mark how they would trim them to make them the same size as $X_{4}$. As each piece is rectangular, assume the trimmings are marked from the top edge, so that a person receives the piece below her mark. See FIGURE 2. Hence, we can speak of one mark as being higher than another. The following procedure will yield an envy-free chore division (we've already described Step 1):

\section{A FOUR-PERSON ENVY-FREE CHORE-DIVISION PROCEDURE}

Step 1. Let Alice and Betty use three applications of Austin's procedure (6 cuts) to obtain 4 pieces $\left(X_{1}, X_{2}, X_{3}, X_{4}\right)$ that Alice and Betty believe are exactly equal in size. If Carl and Debbie disagree on which piece is smallest, then allocate the pieces accordingly and end the procedure. Otherwise, call $X_{4}$ the piece that Carl and Debbie agree is smallest.

Step 2. Let Carl and Debbie mark $X_{1}, X_{2}, X_{3}$ where they would cut them to create ties for smallest with $X_{4}$. Without loss of generality, suppose Debbie has more marks higher than Carl's. Trim the pieces at the higher marks ( 3 cuts), and set aside the trimmings. 


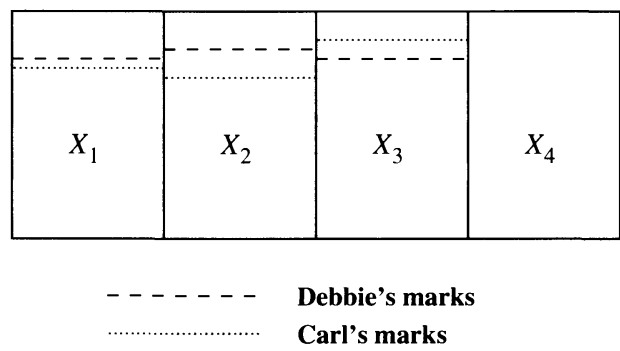

Figure 2 This figure shows a possible set of markings made in Step 1 of our 4-person procedure

Step 3. Let Betty add back to one piece some of the corresponding trimming (1 cut) to create a two-way tie for smallest piece.

Step 4. Let players choose from these pieces in the order Alice-Betty-Carl-Debbie, with Betty required to take the added-back piece if Alice didn't, and Carl required to choose a piece trimmed at his marking if it is available. This will allocate everything except the trimmings in an envy-free fashion.

Step 5. Divide the trimmings (6 cuts), exploiting an irrevocable advantage of Betty over whomever receives $X_{4}$. (The concept of an irrevocable advantage is defined and explained later.) This will allocate the trimmings in an envy-free fashion.

We now verify that this yields an envy-free solution. We assumed in Step 2 that Debbie has more higher marks than Carl (if not, just reverse the roles of Carl and Debbie in what follows). This produces two cases, the first in which Debbie has three higher marks and the second in which Debbie has two.

Case I: Debbie has three higher marks. Assume that Debbie's marks are all at or above Carl's marks. Following Step 2, let Debbie trim $X_{1}, X_{2}$, and $X_{3}$ at her marks to obtain a four-way tie for the smallest piece. Call the trimmed pieces $X_{1}^{\prime}, X_{2}^{\prime}$, and $X_{3}^{\prime}$, and the trimmings $T_{1}, T_{2}$, and $T_{3}$, which are set aside for later. At this point, each person thinks:

$$
\begin{aligned}
\text { Alice: } & X_{1}^{\prime}, X_{2}^{\prime}, X_{3}^{\prime}<X_{4} \\
\text { Betty: } & X_{1}^{\prime}, X_{2}^{\prime}, X_{3}^{\prime}<X_{4} \\
\text { Carl: } & X_{4} \leq X_{1}^{\prime}, X_{2}^{\prime}, X_{3}^{\prime} \\
\text { Debbie: } & X_{1}^{\prime}=X_{2}^{\prime}=X_{3}^{\prime}=X_{4} .
\end{aligned}
$$

Carl must believe $X_{4}$ is the smallest, or tied for the smallest, because his marks were all at or below Debbie's (meaning he believes more should be trimmed to make them equal to $X_{4}$ ).

Of the remaining pieces $X_{1}^{\prime}, X_{2}^{\prime}, X_{3}^{\prime}$, suppose without loss of generality that Betty believes $X_{3}^{\prime} \leq X_{2}^{\prime} \leq X_{1}^{\prime}$. Following Step 3, let Betty return some of the trimmings $T_{3}$ to $X_{3}^{\prime}$ to create a two-way tie for the smallest piece. (We still call the modified piece $X_{3}^{\prime}$, and its trimmings $T_{3}$.) Thus player valuations change:

$$
\begin{aligned}
\text { Alice: } & X_{1}^{\prime}, X_{2}^{\prime}, X_{3}^{\prime}<X_{4} \\
\text { Betty: } & X_{2}^{\prime}=X_{3}^{\prime} \leq X_{1}^{\prime}<X_{4} \\
\text { Carl: } & X_{4} \leq X_{1}^{\prime}, X_{2}^{\prime}, X_{3}^{\prime} \\
\text { Debbie: } & X_{1}^{\prime}=X_{2}^{\prime}=X_{4}<X_{3}^{\prime} .
\end{aligned}
$$


Following Step 4, Alice chooses first (hence is envy-free), then Betty, who is required to take $X_{3}^{\prime}$ if it was not chosen by Alice. Since Betty has at least one of $X_{2}^{\prime}, X_{3}^{\prime}$ to choose from, she is envy-free. Then Carl chooses, and will clearly still have his smallest piece $X_{4}$ available. Debbie will have one of her three smallest pieces available because Betty took $X_{3}^{\prime}$ if Alice did not. Thus all are non-envious of the portions they have recieved so far.

Dividing the Trimmings. The trimmings still need to be divided and assigned. Without loss of generality, suppose that Betty chose $X_{3}^{\prime}$ in the procedure above. Then Betty thinks

$$
\text { Betty: } \quad T_{1}, T_{2} \leq T_{3} \text {. }
$$

Note that because Betty believed $X_{3}=X_{4}$, she could receive all of $T_{3}$ and still not envy Carl. In fact, by the above inequality she could receive $\frac{1}{3}\left(T_{1}+T_{2}+T_{3}\right) \leq T_{3}$ and still not envy him. We will say that Betty has an irrevocable advantage over Carl with respect to the trimmings.

So, lump all the trimmings together (say, $T=T_{1}+T_{2}+T_{3}$ ), and let Alice and Debbie use Austin's procedure to divide $T$ into four pieces that they both agree are all equal. Then let the players choose in the order Carl, Betty, and then (in any order) Debbie and Alice.

With respect to the trimmings, Carl will envy no one because he chooses first. Betty, choosing the smallest of the remaining three pieces, will have a piece that she believes is at most $\frac{1}{3} T$ and therefore will not envy Carl. Alice and Debbie will not envy Betty or Carl because they think all four pieces are equal. Thus the trimmings can be divided in an envy-free fashion.

Case II: Debbie has two higher marks. Assume now that Debbie has two marks at or above Carl's marks. Without loss of generality suppose that Carl has a higher mark on $X_{3}$ than Debbie, as in Figure 2. Following Step 2, let cuts be made at all three highest marks. Then

$$
\begin{aligned}
\text { Alice: } & X_{1}^{\prime}, X_{2}^{\prime}, X_{3}^{\prime}<X_{4} \\
\text { Betty: } & X_{1}^{\prime}, X_{2}^{\prime}, X_{3}^{\prime}<X_{4} \\
\text { Carl: } & X_{3}^{\prime}=X_{4} \leq X_{1}^{\prime}, X_{2}^{\prime} \\
\text { Debbie: } & X_{1}^{\prime}=X_{2}^{\prime}=X_{4}<X_{3}^{\prime} .
\end{aligned}
$$

Following Step 3, let Betty create a two-way tie for the smallest piece (as before) by returning to the smallest piece some of the corresponding trimmings. She may add either to $X_{1}^{\prime}, X_{2}^{\prime}$ or $X_{3}^{\prime}$. The $X_{1}^{\prime}$ and $X_{2}^{\prime}$ cases are equivalent, so we have two subcases.

Suppose Betty adds to $X_{3}^{\prime}$ until it is as large as, say, $X_{2}^{\prime}$. Then

$$
\begin{aligned}
\text { Alice: } & X_{1}^{\prime}, X_{2}^{\prime}, X_{3}^{\prime}<X_{4} \\
\text { Betty: } & X_{2}^{\prime}=X_{3}^{\prime} \leq X_{1}^{\prime}<X_{4} \\
\text { Carl: } & X_{4} \leq X_{1}^{\prime}, X_{2}^{\prime}, X_{3}^{\prime} \\
\text { Debbie: } & X_{1}^{\prime}=X_{2}^{\prime}=X_{4} \leq X_{3}^{\prime} .
\end{aligned}
$$

These inequalities are identical to those in Case I, and thus our procedure works in the same way. Moreover, the trimmings can be handled just as before, since Betty has an irrevocable advantage over Carl (who receives $X_{4}$ ).

Otherwise, suppose Betty adds to $X_{1}^{\prime}$ until it is as large as, say, $X_{2}^{\prime}$ (the $X_{3}^{\prime}$ case is similar). Then 


$$
\begin{aligned}
\text { Alice: } & X_{1}^{\prime}, X_{2}^{\prime}, X_{3}^{\prime}<X_{4} \\
\text { Betty: } & X_{1}^{\prime}=X_{2}^{\prime} \leq X_{3}^{\prime}<X_{4} \\
\text { Carl: } & X_{3}^{\prime}=X_{4} \leq X_{1}^{\prime}, X_{2}^{\prime} \\
\text { Debbie: } & X_{2}^{\prime}=X_{4} \leq X_{1}^{\prime}, X_{3}^{\prime} .
\end{aligned}
$$

Following Step 4, let Alice choose first. When Betty chooses, she will have one of her two smallest pieces available (and will take $X_{1}^{\prime}$ if available as the procedure requires). Next, the procedure requires Carl to take $X_{3}^{\prime}$ if available (since it was the piece trimmed at his marking) and otherwise he recieves $X_{4}$; either way he believes he has the smallest piece. As $X_{1}^{\prime}$ and $X_{3}^{\prime}$ are allocated by this point, we know that Debbie will receive either $X_{2}^{\prime}$ or $X_{4}$, and hence is also envy-free. For the trimmings, note that Betty has an irrevocable advantage over whomever receives the $X_{4}$ piece, so the trimmings can be divided using the method discussed earlier.

This concludes the verification of envy-freeness for all cases. Note that we could alternately have presented tables for each case that list envy-free assignments for Betty, Carl, and Debbie given what Alice chose first. However, remembering those tables would not be as easy as remembering the steps of our procedure.

A bounded $\boldsymbol{n}$-person procedure? Our procedure gives the first known bounded procedure for 4-person envy-free chore division, requiring at most 16 cuts. Actually, this can be reduced to 15 cuts with a modification much like Brams-Taylor-Zwicker's 5-cut modification [5] of the triple application of Austin's procedure.

Although the reader may be tempted to try to further reduce the number of cuts needed for 4-person envy-free chore division, progress in this direction is not as important as the more compelling problem of finding any bounded procedure for more than 4 players. While there do exist finite $n$-person envy-free chore-division procedures ([2], [7]), these are not bounded in the number of steps or cuts, that is, depending on player preferences, they could take arbitrarily long to resolve. For cake-cutting as well as chore division, the existence of bounded $n$-person envy-free division procedures remains a major unsolved problem that will probably require new techniques.

Acknowledgment. The authors gratefully acknowledge a Harvey Mudd College Beckman Research Award, which supports research by undergraduates with faculty.

\section{REFERENCES}

1. A. K. Austin, Sharing a cake, Mathematical Gazette 6:437 (1982), 212-215.

2. S. J. Brams and A. D. Taylor, An envy-free cake division algorithm, Economic Research Reports, C. V. Starr Center for Applied Economics, New York University, 1992.

3. S. J. Brams and A. D. Taylor, An envy-free cake division protocol, Amer. Math. Monthly 102:1 (1995), 9-18.

4. S. J. Brams and A. D. Taylor, Fair Division: From Cake-Cutting to Dispute Resolution, Cambridge University Press, 1996.

5. S. J. Brams, A. D. Taylor, and W. S. Zwicker, A moving-knife solution to the four-person envy-free cakedivision problem, Proc. Amer. Math. Soc. 125 (1997), 547-554.

6. M. Gardner, aha! Insight, W. F. Freeman and Co., New York, 1978.

7. E. Peterson and F. E. Su, Exact procedures for envy-free chore division, HMC Mathematics Department technical report, http://www.math.hmc.edu//su/papers.html, 1998.

8. J. M. Robertson and W. A. Webb, Cake-Cutting Algorithms: Be Fair If You Can, A. K. Peters Ltd., 1998.

9. F. W. Simmons, personal communication, 1998.

10. H. Steinhaus, The problem of fair division, Econometrica 16:1 (1948), 101-104.

11. W. Stromquist, How to cut a cake fairly, Amer. Math. Monthly, 87 (1980), 640-644.

12. F. E. Su, Rental harmony: Sperner's lemma in fair division, Amer. Math. Monthly, 106 (1999), 930-942. 